\title{
Serologic testing for symptomatic coccidioidomycosis in immunocompetent and immunosuppressed hosts
}

\author{
Janis E. Blair ${ }^{1}$, Brandon Coakley ${ }^{1}$, Ana C. Santelli ${ }^{1}$, Joseph G. Hentz ${ }^{2}$ \\ \& Nancy L. Wengenack ${ }^{3}$ \\ ${ }^{1}$ Division of Infectious Diseases, Mayo Clinic, 13400 East Shea Boulevard, Scottsdale, Arizona, AZ 85259, \\ USA; ${ }^{2}$ Biostatistics Unit, Mayo Clinic Scottsdale, Scottsdale, Arizona, USA; ${ }^{3}$ Division of Clinical \\ Microbiology, Mayo Clinic, Rochester, Minnesota., USA
}

Received 25 April 2006; accepted in revised form 17 August 2006

\begin{abstract}
Serologic studies are an important diagnostic tool in the clinical evaluation and follow-up of persons with coccidioidomycosis. Numerous types of serologic tests are available, including immunodiffusion, enzyme immunoassay, and complement fixation. We conducted a retrospective review of the results of 1,797 serologic tests spanning 12 months from the onset of coccidioidomycosis in 298 immunocompetent and 62 immunosuppressed persons with symptomatic infection. Using the onset of symptoms as a reference point, we plotted the positive or negative serologic results over time for both groups. Compared with the immunocompetent group, immunosuppressed persons had lower rates of seropositivity for every type of test during the first year after onset of symptoms for coccidioidomycosis, although many results did not achieve statistical significance. Combining the results of these tests increased the sensitivity of the serologic evaluation in immunocompromised patients. Immunosuppressed persons have the ability to mount a serologic response to coccidioidomycosis, but in some circumstances, multiple methods may be required to improve detection.
\end{abstract}

Key words: coccidioidomycosis, complement fixation, enzyme immunoassay, immunocompetence, immunodiffusion, immunosuppression

Abbreviations: CF - complement fixation; EIA - enzyme immunoassay; ID - immunodiffusion

\section{Introduction}

Coccidioidomycosis is an endemic fungal infection caused by the Coccidioides species C. immitis [1] and the recently described, non-California species C. posadasii [2]. About $60 \%$ of persons with these infections are asymptomatic, and most of the rest have self-limited pulmonary infections, although a minority of cases in otherwise healthy persons may be severe or disseminated [1].

Serologic tests have been used for decades to assist in the diagnosis and management of coccidioidal infection. Among serologic tests available for the diagnosis of mycotic illnesses, those used for coccidioidomycosis are among the most reliable $[3,4]$. At least 7 different serologic methods have been discussed elsewhere [3,5]. Tests commonly used in our endemic area include complement fixation (CF) and immunodiffusion (ID) tests of either tube precipitin antibodies (IgM) or complement-fixing antibodies (IgG). CF or ID tests are often performed in reference laboratories. In addition, an enzyme immunoassay (EIA) to detect $\operatorname{IgG}$ and $\operatorname{IgM}$ can be performed in local clinical laboratories and can often be confirmed by $\mathrm{CF}$ or ID when positive. The EIA for IgG appears 
comparable in sensitivity to CF or ID [6], but the EIA for IgM may give false-positive results and should be confirmed with other serologic methods.

Some authors have demonstrated lower rates of seropositivity in immunosuppressed persons [7-9], whereas others have reported evidence that the serologic response is maintained in immunocompromised hosts [5,10]; most of these observations were seen in small case series, had no comparison group, or contained no temporal information. In contrast, our report reviews our experience with each of the 3 serologic methods in both immunocompetent and immunosuppressed persons as a function of time since onset of illness.

\section{Materials and methods}

\section{Chart review}

A retrospective chart review was conducted for all patients who had coccidioidomycosis diagnosed between January 1, 1999, and October 31, 2003, at our tertiary-care academic medical institution. Patients were identified by an institutional computer search using ICD-9 (International Classification of Diseases, 9th Revision) diagnosis codes and by a manual search of the reports made to the Arizona Department of Health Services. Patients were included only if their records contained adequate documentation of symptoms, laboratory and microbiologic reports, and pathologic findings of coccidioidomycosis. This study was approved by the Mayo Foundation Institutional Review Board.

Abstracted information included age, sex, race, comorbid conditions, dates and results of serologic tests for coccidioidomycosis, microbiologic tests, radiographs, and histopathologic tests. Abstracted information about the clinical coccidioidal syndrome included symptoms, location of infection, treatment, and follow-up.

\section{Definitions}

A patient was defined as having confirmed coccidioidomycosis if $C$. immitis was isolated from the culture of any specimen or if any histopathologic test revealed spherules of Coccidioides spp. Probable coccidioidomycosis was diagnosed when a patient had compatible symptoms (e.g., fever, cough, headache, rash, myalgia, or arthralgia) in association with compatible radiographic changes and positive serologic findings.

Intrathoracic coccidioidomycosis infections involved any of the following tissues: lung, pleura, chest wall, or pericardium. Disseminated coccidioidomycosis required a positive culture or positive histopathologic finding from a specimen outside the thoracic cavity.

A patient was considered to be immunocompromised if any of the following comorbid conditions was present: infection with the human immunodeficiency virus, solid organ or hematologic transplant, hematologic malignancy, or immunosuppression resulting from treatment with corticosteroids, chemotherapy, or other immunosuppressant medications.

\section{Coccidioidal serologic tests}

Several coccidioidal serologic tests were conducted. Our local laboratory performed the EIA to detect IgM and $\mathrm{IgG}$ antibodies using a kit from Meridian Bioscience, Inc (Cincinnati, Ohio). Positive, indeterminate, and negative results were defined according to the manufacturer's instructions (negative $=$ absorbance value $<0.150$; indeterminate $=$ absorbance value $\geq 0.150$ but $\leq 0.199$; or positive $=$ absorbance value $\geq 0.200$ ).

Serum was also sent to the Mayo Clinic Infectious Diseases Serology Laboratory, Rochester, Minnesota, to perform the CF and ID tests. The Laboratory Branch CF test of the Centers for Disease Control and Prevention was used to detect IgG antibodies; it has been described previously [3,11]. As part of this protocol, serum is concentrated in a standardized fashion. The antigen for the CF test was obtained from Dr D. Pappagianis at the University of California at Davis. Between January 1, 1999, and May 30, 2002, the ID test was performed using a kit from Gibson Laboratories, Inc (Lexington, Kentucky) that used antigen $F$ to detect $\operatorname{IgG}$ antibodies. Beginning in June 2002, the ID test was performed using a test kit (Meridian Bioscience, Inc) to detect both TP (early IgM) antibodies and F (late $\mathrm{IgG}$ ) antibodies. To consistently pool the pre- and post-2002 ID results, we excluded the ID IgM results obtained after June 2002 from this analysis. Occasionally, serum samples were sent 
to the external laboratory of D. Pappagianis for serologic evaluation and confirmation of results.

If a patient manifested an anticomplementary $\mathrm{CF}$ result, the $\mathrm{CF}$ test was excluded, although concurrent ID and EIA results were still included in the analysis. On occasion, an indeterminate EIA result was identified and counted as negative. To minimize potential false-positive results, we counted a positive EIA IgM as seropositive only in the presence of another positive result (either EIA $\mathrm{IgG}, \mathrm{ID}, \mathrm{CF}$, or positive microbiologic or histopathologic findings).

\section{Analysis and statistics}

Each serologic result was entered as positive or negative in 1 of many time intervals from the onset of symptoms (weeks 1 through 8 , and months 2 through 12). Individual patients were counted only once in any particular interval and were considered positive for that interval if any serum sample was positive during that period. Patients without serologic testing were not included in this analysis. The percentage of positive assays for each period was calculated from the number of positive samples divided by the number of samples submitted at each interval. Results were graphed as the percentage of positive tests over time. Confidence intervals were calculated for each result in each time block.

Demographic characteristics were compared between immunocompromised and nonimmunocompromised patients using a $\chi^{2}$ test for categorical variables and a 2 -sample $t$ test for continuous variables. Categorical variables are summarized as number (percentage; 95\% normal binomial confidence interval); continuous variables are presented as mean $\pm \mathrm{SD}$ and as median (range). In all cases, a $P$ value of less than .05 is considered statistically significant.

Most of the curves for the qualitative EIA and ID were generated by numerous patients tested in 1 or 2 time periods, and a smaller number of patients had multiple studies performed during the course of a year. The CF curve is a composite of patients receiving serial determinations of this assay to assess for serologic response to infection and treatment. $P$ values for differences were calculated using the Pearson $\chi^{2}$ test.

\section{Results}

Between January 1, 1999, and October 31, 2003, 360 patients had symptomatic, confirmed, or probable coccidioidomycosis with an identifiable onset of symptoms. Of these patients, 298 were immunocompetent and 62 were immunocompromised. Table 1 summarizes the characteristics of these patients and their coccidioidal variables. These 360 patients had a total of 1,797 serologic tests performed in our laboratory, and 314 of the 360 patients had serologic studies within the first year of symptom onset.

The Figure depicts the temporal sequence curves for EIA, CF, ID, and all tests combined for these patients. It compares the seroreactivity among immunocompromised and immunocompetent hosts.

Table 2 summarizes the serologic results for EIA, CF, and ID among the different groups of immunocompromised hosts. These results are also compared with those for 252 immunocompetent patients who were tested within the first year of symptom onset. In the immunosuppressed group, no single test was $100 \%$ sensitive for all groups, although the CF test was $100 \%$ sensitive for the "other immunocompromised" group (10 positive of 10 tested). This same test was not sensitive for organ transplantation ( 2 positive of 6 tested), and it had an overall sensitivity of only 67\% (35 of 52) (Fig. 1).

\section{Discussion}

Immune competence plays a clear role in the clinical course and control of coccidioidomycosis, initially with innate immunity and subsequently with adaptive immunity afforded by the $\mathrm{T}_{\mathrm{H}} 1$ mediated immune response [12]. Numerous studies have demonstrated an increased likelihood of severe or disseminated infection among persons with compromised immunity $[7,10,13]$.

The diagnosis of coccidioidomycosis can be challenging, whether patients are immunocompetent or immunosuppressed. Typical symptoms and radiographic findings are often nonspecific and resemble pulmonary infection from other organisms. Many patients with coccidioidomycosis have a nonproductive cough, and sputum samples can be difficult to obtain for culture without an invasive 
Table 1. Characteristics of 360 immunosuppressed and immunocompetent patients with symptomatic coccidioidomycosis

\begin{tabular}{|c|c|c|c|c|c|}
\hline \multirow[t]{2}{*}{ Variable } & \multicolumn{2}{|c|}{$\mathrm{ICH}(N=62)$} & \multicolumn{2}{|c|}{ Non-ICH $(N=298)$} & \multirow[t]{2}{*}{$P$ value } \\
\hline & No. $(\%)$ & $95 \% \mathrm{CI}$ & No. $(\%)$ & $95 \% \mathrm{CI}$ & \\
\hline \multicolumn{6}{|l|}{ Patient characteristics } \\
\hline Age, y (mean \pm SD) & $64.8 \pm 13.9$ & & $56.8 \pm 16.2$ & & $<.001$ \\
\hline Male sex & $39(63)$ & $51-75$ & $157(53)$ & $47-58$ & .14 \\
\hline White race & $57(92)$ & $85-99$ & $238(80)$ & $75-84$ & .03 \\
\hline \multicolumn{6}{|l|}{ Patient medical history } \\
\hline Cancer & $33(53)$ & $41-66$ & $60(20)$ & $16-25$ & $<.001$ \\
\hline Cardiovascular disease & $36(58)$ & $46-70$ & $120(40)$ & $35-46$ & .01 \\
\hline Tobacco use & $34(55)$ & $42-67$ & $156(52)$ & $47-58$ & .72 \\
\hline Diabetes mellitus & $15(24)$ & $14-35$ & $40(13)$ & $10-17$ & .03 \\
\hline HIV infection & $4(6)$ & $0-13$ & $0(0)$ & $0(0)$ & $<.001$ \\
\hline Rheumatologic illness & $13(21)$ & $11-31$ & $20(7)$ & $4-10$ & $<.001$ \\
\hline Organ transplantation & $7(11)$ & $3-19$ & $0(0)$ & $0(0)$ & $<.001$ \\
\hline Hematologic malignancy & $14(23)$ & $12-33$ & $0(0)$ & $0(0)$ & $<.001$ \\
\hline Nonhematologic malignancy & $19(31)$ & $19-42$ & $68(23)$ & $18-28$ & .3 \\
\hline \multicolumn{6}{|l|}{ Coccidioidal illness } \\
\hline Lungs only & $39(63)$ & $51-75$ & $231(78)$ & $73-82$ & .02 \\
\hline Limited to thoracic cavity & $50(81)$ & $71-90$ & $267(90)$ & $86-93$ & .048 \\
\hline Extrathoracic dissemination & $12(19)$ & $10-29$ & $31(10)$ & $7-14$ & .048 \\
\hline Confirmed by positive microbiologic or histologic tests & $32(52)$ & $39-64$ & $80(27)$ & $22-32$ & $<.001$ \\
\hline
\end{tabular}

$\mathrm{CI}$, confidence interval; ICH, immunocompromised; non-ICH, nonimmunocompromised; HIV, human immunodeficiency virus.

Table 2. Seropositivity among 62 immunocompromised hosts with serologic confirmation of coccidioidomycosis detected by various serologic tests

\begin{tabular}{|c|c|c|c|c|c|c|c|c|}
\hline \multirow[t]{3}{*}{ Category of immunosuppression } & \multicolumn{8}{|c|}{ Type of serologic testing, no. $(\%)$} \\
\hline & \multicolumn{2}{|c|}{$\begin{array}{l}\text { EIA (IgM } \\
\text { and } \operatorname{IgG})\end{array}$} & \multicolumn{2}{|l|}{$\mathrm{CF}$} & \multicolumn{2}{|c|}{$\begin{array}{l}\text { ID }(\operatorname{IgM} \text { or } \\
\text { IgG or both) }\end{array}$} & \multicolumn{2}{|c|}{ Any test } \\
\hline & Tested & Positive & Tested & Positive & Tested & Positive & Tested & Positive \\
\hline Hematologic malignancy $(N=14)$ & 12 & $4(33)$ & 10 & $6(60)$ & 6 & $1(17)$ & 12 & $8(67)$ \\
\hline Cancer and chemotherapy, nonhematologic $(N=19)$ & 18 & $13(72)$ & 18 & $12(67)$ & 15 & $9(60)$ & 19 & $18(95)$ \\
\hline HIV infection $(N=4)$ & 4 & $1(25)$ & 3 & $2(67)$ & 3 & $2(67)$ & 4 & $3(75)$ \\
\hline Organ transplantation $(N=7)$ & 7 & $5(71)$ & 6 & $2(33)$ & 3 & $0(0)$ & 7 & $5(71)$ \\
\hline Rheumatologic illness $(N=13)$ & 11 & $9(82)$ & 10 & $6(60)$ & 8 & $4(50)$ & 11 & $10(91)$ \\
\hline Other ICH illness $^{*}(N=11)$ & 10 & $9(90)$ & 10 & $10(100)$ & 8 & $6(75)$ & 10 & $10(100)$ \\
\hline All patients ${ }^{\dagger}$ & 57 & $38(67)$ & 52 & $35(67)$ & 40 & $21(53)$ & 58 & $49(84)$ \\
\hline $\begin{array}{l}\text { Healthy patients tested } \leq 1 \text { y after symptom } \\
\text { onset }(N=261)\end{array}$ & 244 & $212(87)$ & 252 & $188(75)$ & 248 & $180(73)$ & 261 & $247(95)$ \\
\hline
\end{tabular}

CF, complement fixation; EIA, enzyme immunoassay; ICH, immunocompromised; ID, immunodiffusion; HIV, human immunodeficiency virus.

*Patients with other causes of immunocompromise include 3 inflammatory bowel disease (1 taking infliximab), 2 autoimmune blood dyscrasias (hemolytic anemia and idiopathic thrombocytopenic purpura) taking prednisone, 1 autoimmune polyneuropathy, and 5 taking corticosteroids long-term for sarcoid, cough, other pulmonary diseases (chronic obstructive pulmonary disease, interstitial pulmonary fibrosis, or normal interstitial pneumonia).

${ }^{\dagger}$ Six patients have 2 immunosuppressive illnesses and are represented in each category.

procedure. A histopathologic diagnosis generally requires tissue obtained by an invasive procedure. Newer diagnostic methods under development include polymerase chain reactions [14] and assays to assess the cellular immune response to coccidioidomycosis [15]; the ultimate utility of these tests in the everyday diagnosis of this infection is not clear at this time. 

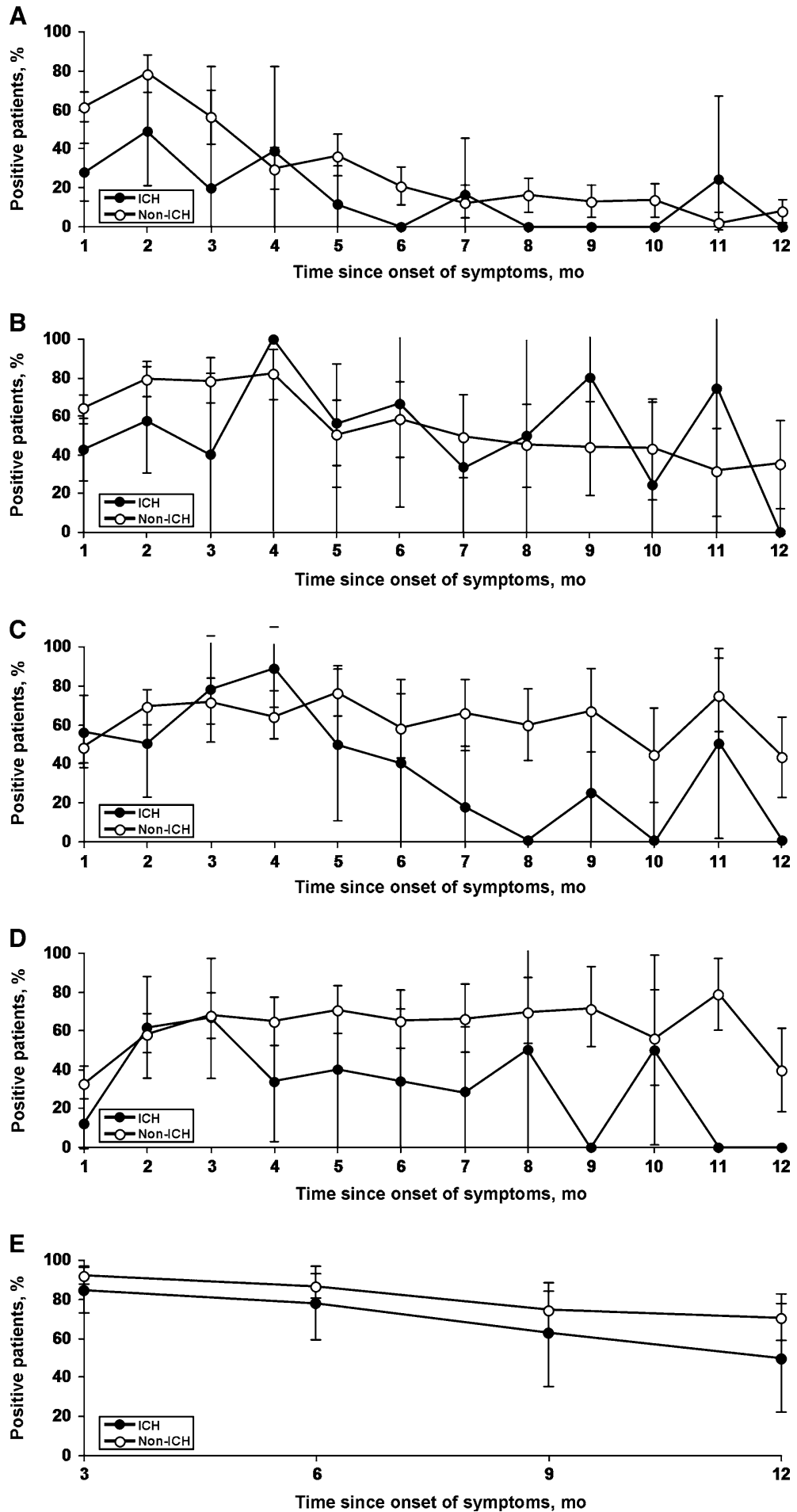

Figure 1. Temporal sequence of serologic responses after onset of symptoms in immunocompromised (ICH) versus nonimmunocompromised (non- $\mathrm{ICH}$ ) patients with symptomatic coccidioidomycosis. Percentage of patients positive for coccidioidomycosis, $\mathbf{A}$, by enzyme immunoassay for IgM; B, by enzyme immunoassay for IgG; C, by complement fixation; D, by immunodiffusion; and, E, by any test. 
Serologic tests therefore play an important role in the diagnosis and follow-up of patients with coccidioidomycosis. For decades, assays have been used to detect early IgM antibodies and later IgG antibodies. Although many assay methods have been used in the past, the most commonly used IgM serologic methods now include ID and EIA, whereas commonly used IgG methods include ID, quantitative ID, CF, and EIA. Some authors have reported a good correlation of the EIA with more traditional assays [16]; others have noted potential problems with specificity and have emphasized the need for confirmation by ID [6]. The EIA and ID assays used in our laboratories are qualitative tests, whereas the $\mathrm{CF}$ is a quantitative test that is useful in following the course of illness over time; quantitative results are best interpreted when specimens from different time points are analyzed simultaneously [5]. Some reference laboratories offer a quantitative ID (IDCF) for late IgG, which also allows a quantitative result. Titer results may vary from laboratory to laboratory, which can hinder interpretation; therefore, quantitative studies performed serially are best interpreted if performed by the same laboratory.

It is important to note that the sensitivity of any assay in either compromised or healthy persons is modest at best, regardless of when the patient is tested relative to the onset of symptoms. When serologic studies are so heavily relied upon to make a diagnosis, negative studies can delay diagnosis, result in the need for invasive testing, and delay initiation of therapy. Since all our patients had confirmed or probable coccidioidomycosis, specificity of the test is not addressed in this study.

In a few situations, the sensitivity of the EIA appears to be greater than that of the CF or ID tests, raising some concern that these cases represent false-positive findings. However, when we analyzed the subgroup of patients whose diagnosis of coccidioidomycosis was confirmed with positive histopathologic or microbiologic results, the relative positivity among the various serologic methods did not change (data not shown).

Although the magnitude of the finding is unclear, previous authors have observed a decreased serologic response in immunocompromised hosts who contract coccidioidomycosis $[3,7-9,17]$. Some of these studies examined 1 or 2 methods performed in reference laboratories.
Other authors reported the ability of immunocompromised hosts to mount an adequate serologic response by $\mathrm{CF}$, but the height of the titer may be blunted when compared with that of immunocompetent patients [10]. Our study evaluated 3 different methods, a locally performed EIA and a CF and ID sent to an external reference laboratory. For the present study, serum samples for $\mathrm{CF}$ were sent to 1 of 2 different reference laboratories even for individual patients during their course of illness, which made the quantitative differences between immunocompetent and immunocompromised persons difficult to interpret; we subsequently opted not to pursue this line of evaluation.

Our study summarized the seroreactivity of patients as a function of time since symptom onset and compared the results of various serologic tests between immunocompetent patients and immunosuppressed hosts. The curves for the qualitative EIA and ID are composite curves generated by numerous patients tested in 1 or more time periods over the course of a year; on the basis of our observations in this study, we agree with other authors that there is often little to be gained by repeating a positive qualitative test [5]. The CF curve is a composite of patients receiving serial determinations of this assay to assess for a serologic response to infection or treatment. In nearly every comparison time point, immunocompetent patients had a higher overall rate of seropositivity for each of the different serologic methods but only a few data points achieved statistical significance. This finding likely reflects either no (or small) difference between the groups or small or heterogeneous cohorts or both. Even when all the test results were combined, we did not find a significantly decreased rate of seropositivity in the immunocompromised group. In fact, there appeared to be a higher rate of seropositivity when all methods were considered than when each test was considered alone. In light of the increased sensitivity of a panel of tests compared with any single test, it may be prudent to test immunocompromised patients by multiple methods before concluding that a patient is seronegative. A long-established method for improving the yield of serologic testing has been to concentrate serum before the test, which is recommended [5] and practiced in the 2 reference laboratories we use. 
Subgroups of the immunocompromised patients had differences in seropositivity, ranging from those with hematologic malignancy (overall seropositivity, $69 \%$ ) to a $100 \%$ seropositive rate in the miscellaneous group of immunosuppressed patients. This observation most likely reflects inherent differences in the immune capabilities of each group, but the groups were too small to enable us to draw definitive conclusions.

Weaknesses of this study were its retrospective nature and tertiary-care bias. This study did not attempt to correlate the laboratory results to the clinical course of patients. Many patients were evaluated by numerous physicians, and the number and choices of serologic studies were those deemed appropriate on a case-by-case basis. The time of the patients' initial evaluation at our medical center in relation to the onset of their symptoms varied from a few days to several months. Serum samples were taken at various times during the patient's illness, and there was no uniformity in the types of serologic tests ordered by different physicians. Therefore, each point in the time line may represent a different cohort of patients and simply reflect the positivity of the test at that particular time for the patients tested. A large prospective study of serologic evaluations over time would address some of these issues. The referral center bias is evident in the number of extrapulmonary infections observed in the healthy population as well as by the large percentage of biopsy- or culture-proven infections.

Inherent problems also exist when patients with so many different types of immunosuppressant illnesses or treatments are grouped into a single cohort. In this study, the many different illnesses and their immunosuppressant-inducing treatments included hematologic malignancies, cancer chemotherapy, organ or stem cell transplantation, human immunodeficiency virus infection, and numerous systemic illnesses (such as connective tissue diseases) requiring therapy with immune modifiers. These patients may have had various immune defects, some of which may or may not be important in the detection or control of coccidioidomycosis. In addition, some patients (e.g., organ transplant recipients) may have varying levels of immunosuppression throughout the course of the illness, with marked immune impairment early on and less impairment over time. None of these differences was taken into account in the analysis of the immunocompromised group in this study, and these differences may be an important barrier to detection of significant differences in seroreactivity. In addition, the immunocompromised group was older and had more comorbid illness than the noncompromised cohort, factors that again impede comparison of the 2 groups.

In summary, our evaluation of coccidioidal serologic tests among 360 immunocompetent and immunosuppressed patients using 1,797 serologic samples showed that, for any single serologic test, immunocompetent hosts appeared to be more likely than immunosuppressed patients to be seropositive with active coccidioidomycosis. When the test results from 3 different methods were considered as a whole, the sensitivity of the serologic evaluation increased in the immunocompromised patients, but this overall result was not statistically different for immunosuppressed versus immunocompetent persons. Immunosuppressed persons have the ability to mount a serologic response to coccidioidomycosis, but multiple methods may be required to detect that seropositivity. Future prospective studies evaluating large homogeneous cohorts may further clarify these results.

\section{Acknowledgment}

Statistical assistance was provided by the Center for Patient Oriented Research, Mayo Clinic.

Editing, proofreading, and reference verification were provided by the Section of Scientific Publications, Mayo Clinic.

\section{References}

1. Chiller TM, Galgiani JN, Stevens DA. Coccidioidomycosis. Infect Dis Clin North Am 2003; 17: 41-57.

2. Fisher MC, Koenig GL, White TJ, Taylor JW. Molecular and phenotypic description of Coccidioides posadasii sp. nov., previously recognized as the non-California population of Coccidioides immitis. Mycologia 2002; 94: 73-84.

3. Pappagianis D, Zimmer BL. Serology of coccidioidomycosis. Clin Microbiol Rev 1990; 3: 247-268.

4. Smith CE, Saito MT, Simons SA. Pattern of 39,500 serologic tests in coccidioidomycosis. JAMA 1956; 160: 546-552.

5. Pappagianis D. Serologic studies in coccidioidomycosis. Semin Respir Infect 2001; 16: 242-250. 
6. Kaufman L, Sekhon AS, Moledina N, Jalbert M, Pappagianis D. Comparative evaluation of commercial Premier EIA and microimmunodiffusion and complement fixation tests for Coccidioides immitis antibodies. J Clin Microbiol 1995; 33: 618-619.

7. Blair JE, Logan JL. Coccidioidomycosis in solid organ transplantation. Clin Infect Dis. 2001 Nov 1; 33: 1536-44. Epub 2001 Oct 4.

8. Rutala PJ, Smith JW. Coccidioidomycosis in potentially compromised hosts: the effect of immunosuppressive therapy in dissemination. Am J Med Sci 1978; 275: 283295.

9. Torres HA, Rivero GA, Kontoyiannis DP. Endemic mycoses in a cancer hospital. Medicine (Baltimore) 2002; 81: 201-12.

10. Deresinski SC, Stevens DA. Coccidioidomycosis in compromised hosts: experience at Stanford University Hospital. Medicine (Baltimore) 1975; 54: 377-395.

11. Palmer DF, Kaufman L, Kaplan W, Cavallaro JJ. Serodiagnosis of mycotic disease. Springfield, IL: CC Thomas, 1977.

12. Cox RA, Magee DM. Coccidioidomycosis: host response and vaccine development. Clin Microbiol Rev 2004; 17 : 804-839.

13. Fish DG, Ampel NM, Galgiani JN, Dols CL, Kelly PC, Johnson $\mathrm{CH}$ et al. Coccidioidomycosis during human immunodeficiency virus infection: a review of 77 patients. Medicine (Baltimore) 1990; 69: 384-391.

14. Johnson SM, Simmons KA, Pappagianis D. Amplification of coccidioidal DNA in clinical specimens by PCR. J Clin Microbiol 2004; 42: 1982-1985.

15. Ampel NM, Kramer LA, Li L, Carroll DS, Kerekes KM, Johnson SM et al. In vitro whole-blood analysis of cellular immunity in patients with active coccidioidomycosis by using the antigen preparation T27 K. Clin Diagn Lab Immunol 2002; 9: 1039-1043.

16. Martins TB, Jaskowski TD, Mouritsen CL, Hill HR. Comparison of commercially available enzyme immunoassay with traditional serological tests for detection of antibodies to Coccidioides immitis. J Clin Microbiol 1995; 33: 940-943.

17. Pappagianis D. Current and future approaches to the diagnosis of coccidioidomycosis. In: Einstein HE, Catanzaro A, eds. Coccidioidomycosis: Proceedings of the 5th International Conference on Coccidioidomycosis, National Foundation for Infectious Diseases, 1994 Aug 24-27. Stanford, CA, 1996: 116-128.

Address for correspondence: Janis E. Blair, Division of Infectious Diseases, Mayo Clinic, 13400 East Shea Boulevard, Scottsdale, Arizona, AZ 85259, USA 